Article

\title{
A Multi-Physics Adaptive Time Step Coupling Algorithm for Light-Water Reactor Core Transient and Accident Simulation
}

\author{
Alexey Cherezov (D), Jinsu Park, Hanjoo Kim, Jiwon Choe and Deokjung Lee * \\ Department of Nuclear Engineering, Ulsan National Institute of Science and Technology, Ulsan 44919, Korea; \\ alcher@unist.ac.kr (A.C.); ccpj0310@unist.ac.kr (J.P.); hanjookim@unist.ac.kr (H.K.); chi91023@unist.ac.kr (J.C.) \\ * Correspondence: deokjung@unist.ac.kr
}

Received: 22 October 2020; Accepted: 27 November 2020; Published: 2 December 2020

\begin{abstract}
A new reactor core multi-physics system addresses the pellet-to-cladding heat transfer modeling to improve full-core operational transient and accident simulation used for assessment of reactor core nuclear safety. The rigorous modeling of the heat transfer phenomena involves strong interaction between neutron kinetics, thermal-hydraulics and nuclear fuel performance, as well as consideration of the pellet-to-cladding mechanical contact leading to dramatic increase in the gap thermal conductance coefficient. In contrast to core depletion where parameters smoothly depend on fuel burn-up, the core transient is driven by stiff equation associated with rapid variation in the solution and vulnerable to numerical instability for large time step sizes. Therefore, the coupling algorithm dedicated for multi-physics transient must implement adaptive time step and restart capability to achieve prescribed tolerance and to maintain stability of numerical simulation. This requirement is met in the MPCORE (Multi-Physics Core) multi-physics system employing external loose coupling approach to facilitate the coupling procedure due to little modification of constituent modules and due to high transparency of coupling interfaces. The paper investigates the coupling algorithm performance and evaluates the pellet-to-cladding heat transfer effect for the rod ejection accident of a light water reactor core benchmark.
\end{abstract}

Keywords: multi-physics; external loose coupling; rod ejection accident; adaptive time step; picard iteration

\section{Introduction}

Multiphysics analysis attracts a lot of attention of nuclear engineers worldwide as it helps to produce more realistic results in terms of reactor core safety margins. Nowadays there are many codes progressing towards the high-fidelity multiphysics simulation of light water reactors [1,2]. Recent works $[3,4]$ demonstrate the coupling experience of MCS [5], CTF [6] and FRAPCON [7] codes for reactor core pin-by-pin analysis taking into consideration the pellet-to-cladding transfer and coolant cross-flow phenomena. To continue this work, the Computational Reactor Physics and Experiment Laboratory of Ulsan National Institute of Science and Technology (UNIST CORE lab) has developed MPCORE (Multi-Physics CORE) code [8] capable for both depletion and transient analysis of a light-water reactor core with the dynamic pellet-to-cladding heat transfer coefficient taking into account fuel rod irradiation, thermal-mechanics and corrosion phenomena. The new multiphysics system implements the external loose coupling of pin-by-pin neutronics, thermal-hydraulics and fuel performance using the external adaptive time step selection algorithm to achieve the reactor core high-fidelity simulation with prescribed 
tolerance. The processing of the multi-physics data producing by the MPCORE code has been discussed previously [9]. This work is devoted to the application of the adaptive time step integration algorithm, analysis of the code computational performance and evaluation of the pellet-to-cladding heat transfer effect on the safety parameters using for reactor core rod ejection accident (REA) transient [10-12].

MPCORE performs numerical integration of reactor core codes using Implicit Euler step-doubling method $[13,14]$. The time step selection algorithm estimates time discretization error at the current time step and then chooses the next step size in such a way that a given tolerance is not exceeded. For a chosen step size one transient step is calculated with this step size and compared to the result of two transient steps with half the step size. This approach is known as the two-level step-doubling method described in the works [13]. We chose this method as being more robust compared to embedded pair methods such as considered in [14,15]. The implicit equation within a time step is solved by the damped Picard method [14] with Gauss-Seidel acceleration for better convergence.

The current MPCORE version comprises three computational modules: three-dimensional two-group nodal diffusion code RAST-K [16] augmented a pin-by-pin reconstruction module, homogeneous one-phase Coolant Thermal-Hydraulics One Dimensional code CTH1D and Fuel Rod Analysis Programming Interface FRAPI designed for the external loose coupling of fuel rod analysis codes FRAPCON [7] and FRAPTRAN [17]. The description and verification of FRAPI code was presented recently [18].

The paper is organized in five sections. Section 1 is the introduction part. Section 2 describes the pellet-to-cladding heat transfer phenomena, multi-physics system modules and the multi-physics coupling algorithm. Section 3 is devoted to the analysis of the rod ejection accident (REA) from hot zero power (HZP) core, effect of the gap heat transfer coefficient (HTC) on the reactor core safety parameters, and simulation convergence and performance. Section 4 contains the conclusion and future work plan.

\section{Methodology}

\subsection{Pellet-to-Cladding Heat Transfer Phenomena}

The temperature distribution in a fuel pellet, cladding and coolant is of primary importance of multi-physics simulation because it has a great influence on the distribution of neutrons due to the Doppler effect and coolant density change. The heat transfer phenomena inside a fuel rod comprises heat conduction in the fuel pellets and cladding, and the heat transfer across the pellet-cladding gap filled with a gas under high pressure [19]. The pellet heat conduction mainly depends on the heat capacity and thermal conductivity coefficients which are the functions of local fuel burnup, temperature and density. Notwithstanding the influence of the restructuring effects such as fuel cracking, grain growth and high burnup structure formation, the effective fuel Doppler temperature used for neutronics feedback is predicted with enough high accuracy for engineering calculation using the heuristically obtained correlations. In contrary, the simulation of the heat transfer across a gap is a more challenging problem as the pellet-cladding HTC primarily depends on the radial distance between a pellet and cladding surfaces, and, consequently, affected by the thermal-mechanics and fission gas release. Therefore, the rigorous simulation of pellet-to-cladding heat transfer involves synthesis of neutronics, coolant thermal-hydraulics and fuel performance codes.

The pellet-to-cladding heat flux $q_{F}$ in watts per unit area is calculated as follows

$$
q_{F}=h_{g a p}\left(T_{f s}-T_{c i}\right),
$$


where $T_{f_{s}}$ is the outer surface fuel pellet temperature in $K, T_{c i}$ is the inner surface cladding temperature in $K$ and $h_{\text {gap }}$ in $\mathrm{W} / \mathrm{m}^{2} \mathrm{~K}$ is the heat transfer coefficient between fuel and cladding. Overall, the fuel-cladding gap conductance model [20] consists of four parallel conduction routes:

$$
h_{\text {gap }}=h_{\text {solid }}+h_{\text {gas }}+h_{\text {rad }}+h_{\text {con }}
$$

where $h_{g a s}$ is the gas heat conductance, $h_{\text {rad }}$ is the conductance by radiation from fuel outer surface to cladding inner surface, $h_{\text {solid }}$ is the conductance by solid pellet-cladding contact and $h_{\text {con }}$ is the conductance by gas convection which can be neglected as the gas is quiescent and fuel rod radial gap size is very small, about $60 \mu \mathrm{m}$ at the hot state.

The radiation heat conductance term $h_{\text {rad }}$ is described by the Stefan-Boltzmann law

$$
h_{\text {rad }}=\sigma \epsilon\left(T_{f s}^{4}-T_{c i}^{4}\right) /\left(T_{f s}-T_{c i}\right)
$$

where $\sigma$ is the Stefan-Boltzmann constant, $\epsilon$ is the emissivity factor. The thermal conduction by radiation is normally less than $1 \%$ during normal operation condition due to the limited surface temperatures. However, if cladding ballooning occurs, the distance between pellet and cladding surfaces becomes too large and radiation heat transfer can outperform the heat transfer by gas conductance.

The pellet-cladding gap filled with the as-fabricated helium gas acquiring a large thermal conductivity coefficient and gaseous fission products, primarily krypton and xenon, released from uranium dioxide during irradiation. The gas heat conductance is given by the model of Ross and Stoute [21],

$$
h_{g a s}=\frac{\lambda_{g a s}}{\delta_{g a p}+\delta_{f}+\delta_{c}+\delta_{j u m p}}
$$

where the thermal conductivity of a multicomponent gas $\lambda_{\text {gas }}$ is divided by sum of the gap size $\delta_{\text {gap }}$, fuel and cladding mean roughness $\delta_{f}$ and $\delta_{c}$, and combined fuel and cladding gas temperature jump distance $\delta_{\text {jump }}$.

The temperature jump distance, also known as the gas extrapolation length, accounts for the temperature discontinuity near the fuel and cladding walls caused by the accommodation effect. The accommodation effect appears to be important if the gap width is small compared with the gas mean free path and gas molecules travel back and forth between walls rarely colliding with each other. For example, at $20 \mathrm{MPa}$ gas pressure and $900 \mathrm{~K}$ gas temperature the jump distance for helium is about $10 \mu \mathrm{m}$, for xenon about $1 \mu \mathrm{m}$. According to the Maxwell gas-surface interaction model, gas molecules undergo the diffusive reflection associated with the complete thermal accommodation to a wall surface temperature and the specular reflection that does not change the particle's energy. The specular reflection and the inability of gas molecules leaving a surface to fully exchange their energy with neighboring gas molecules results in a steep temperature gradient near the fuel and cladding surfaces which is computationally adjusted by $\delta_{\text {jump }}$ in Equation (4). The detailed gas-surface interaction model, including the fraction of specular reflection events, for different gas and wall materials is the subject of many experimental measurements and theoretical studies [22].

Thermal expansion, cladding creep under external pressure, irradiation effects, fuel relocation due to cracking all contribute to the mechanical deformation of a fuel pellet and cladding, which results in a gradual decrease in the gap width $\delta_{\text {gap }}$ over fuel burn-up until it disappears after 15-20 GWD/MTU [23]. The pellet-cladding mechanical interaction at lower burnups is mainly observed for reactivity initiated accident events accompanied with high temperatures owing to the differential thermal expansion between fuel pellet and cladding. When the fuel pellets come into contact with the cladding, the heat transfer coefficient $h_{\text {gap }}$ dramatically increases owing to thermal conduction through contact spots separated 
by microscopic valleys of the contact area. The solid conductance term $h_{\text {solid }}$ depends on the thermal conductivity of cladding and pellet, interfacial pressure and surface roughness $[17,21]$. Thus, owing to the presence of surface asperities, the heat transferred across the contact interface consists of three components $h_{\text {solid }}, h_{\text {gas }}$ and $h_{\text {rad }}$ with the primary contribution arising from the first term.

\subsection{MPCORE Modules}

MPCORE is designed in such a way that it is able to link any number of external codes using the interface functions demanded from each external module. The current version supports three computational modules:

- RAST-K [16] is a three-dimensional two-group nodal diffusion code used for depletion and transient analysis of light water reactor cores having square assemblies. The nodal solver is accompanied with pin-by-pin power reconstruction, micro-depletion analysis and xenon/samarium build-up modules. The two-group cross-section library and pin-wise power shape functions for the reconstruction module are computed by lattice code STREAM. This allows the fuel assemblies with asymmetric burnable rod position to be assessed [24].

- $\quad$ FRAPI [18] is the fuel rod analysis program interface designed for the loose coupling of the fuel rod analysis codes FRAPCON and FRAPTRAN [17] with other reactor core codes. The interface supports most of the original code options beside the two-dimensional finite element analysis of fuel rod cladding. The diagram of the phenomena simulated by the codes is presented in Figure 1.

- CTH1D is the homogeneous one-phase code for transient and steady-state simulation of coolant flow in a fuel rod channel. The code implements one-dimensional energy and mass conservation equations with the steam-water properties provided by FREESTEAM which is an open source implementation of international-standard IAPWS-IF97. In addition, CTH1D is able to simulate coolant boiling effect based on the correlations of critical heat flux and heat transfer coefficient adopted from FRAPTRAN.

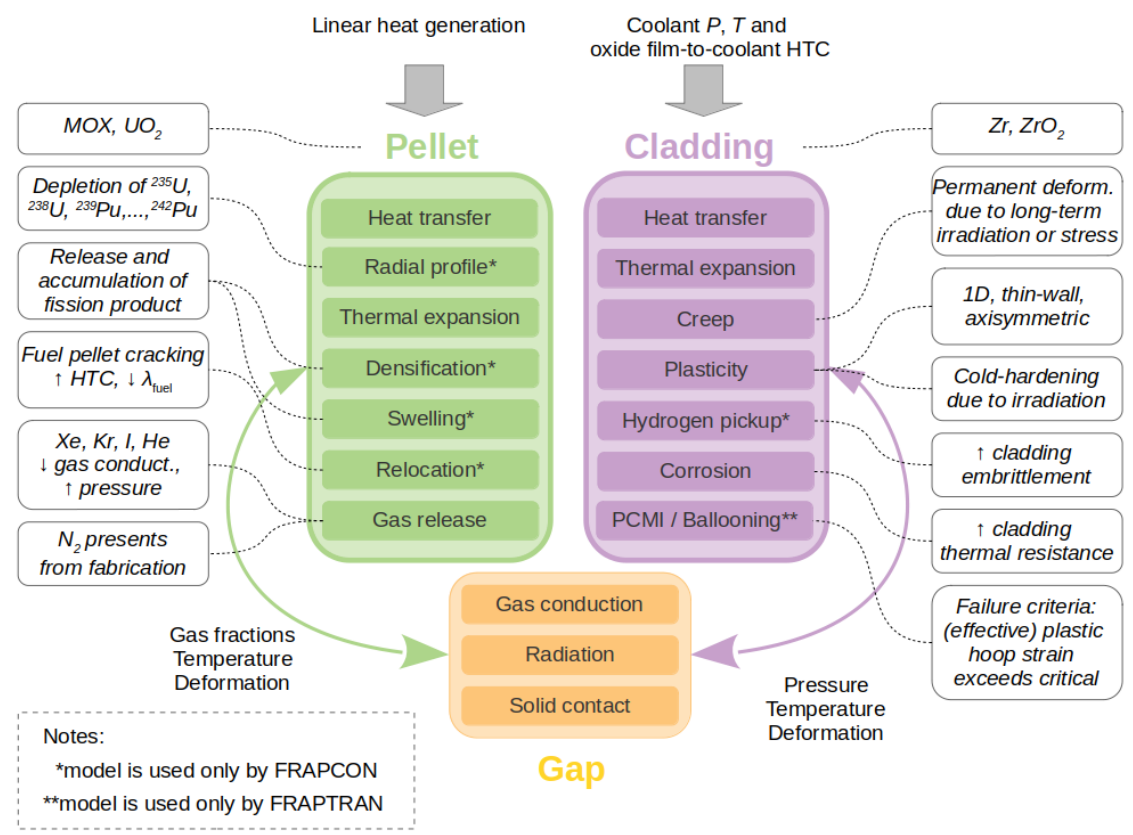

Figure 1. Fuel Rod Analysis Programming Interface (FRAPI) models. 
The feedback exchange between computational modules is implemented according to the scheme presented in Table 1. In detail, RAST-K calculates pin-by-pin linear power distribution $q_{l}$ which is forwarding to FRAPI. The FRAPI code calculates fuel pellet Doppler temperature $T_{\text {fuel }}$, average cladding temperature $T_{\text {clad }}$ and cladding oxide film temperature $T_{f i l m}$, the last two parameters are used as the boundary conditions for coolant thermal-hydraulics. CTH1D calculates bulk coolant temperature $T_{\text {cool }}$, bulk coolant density $D_{\text {cool }}$ and film heat transfer coefficient $H_{\text {film }}$ adopting by fuel rod analysis code FRAPI. Updated $T_{\text {fuel }}, T_{\text {cool }}, D_{\text {cool }}$ and $T_{\text {clad }}$ variables are used by RAST-K for the two-group cross-section calculation. The iteration repeats until the convergence of all the feedback parameters.

\subsection{Integration Scheme}

MPCORE code performs the external loose coupling of multi-physics modules into a united multi-physics system by the medium of routines listed in Table 2. For the interaction with the multi-physics system each module maintains allocation $A$ and deallocation $D$ routines to create and destroy a module's internal memory space; save $S$ and load $L$ routines to memorize and restore a module's state; initial steady-state $I$ and time step marching $N$ routines; update routine $U$ which is used for the feedback vector update.

The feedback vectors $u_{i}$ are located in the external memory space shared by all the multi-physics modules, while the module's state vectors $x_{i}$ are situated in the module's internal memory spaces. This approach is employed as a multi-physics module, which usually presents a complex engineering code where the state depends on the physical variables, flags, counters, and logical descriptors needed for algorithm control. In order to avoid dealing with the code's internal variables and mitigate the profound change of the engineering code we allow the state vectors $x_{i}$ to be unavailable for explicit use. The step-doubling and fixed-point algorithms can be implemented in the subspace of the feedback parameters $u$ without an explicit operation of the corresponding state vector $x_{i}$.

We explain the coupling algorithm in terms of the interface functions presented in Table 2 and using the following notation: " $A B C$ " denotes the successive call of the routines " $A$ ", " $B$ " and " $C$ " from left to right, brackets " $(A B)$ " denote a cyclic run of the routines $A$ and $B$ such that the sequence " $A B A B A B \ldots$ " repeats until the convergence, the multiple $\operatorname{dot}$ " $A_{1} \ldots A_{n}$ " denotes the successive run of routines $A_{1}, A_{2}, \ldots, A_{n}$.

There are many methods for the solution of implicit equations such as Picard iterations, Anderson's acceleration and Jacobi-Free Newton Krylov methods [25]. We implemented the damped Picard iteration method improved by Gauss-Seidel acceleration. The basic Picard iteration algorithm is given by

$$
P_{X}^{\prime}=\left(L_{1} \ldots L_{n} X_{1} \ldots X_{n} U_{1} \ldots U_{n}\right),
$$

where $X$ is the routine of initial steady-state $I$ or time step marching $N$. On every iteration the multiphysics modules return to the original state by calling the subroutines $L_{i}$. Then routines $X_{i}$ update the multiphysics modules states with respect to the feedback vectors $u_{i}$ updated on the previous iteration by routines $U_{i}$. The cycle exits when the convergence for all feedback parameters is achieved.

The convergence rate of the Picard iterations can be improved by the swapping of the routines $X_{i}$ and $U_{i}$ in Equation (5) that results in a Picard iteration with the Gauss-Seidel acceleration, that is

$$
P_{X}=\left(L_{1} \ldots L_{n} X_{1} U_{1} \ldots X_{n} U_{n}\right)
$$

For the time step integration of the coupled dynamical system MPCORE uses the algorithm

$$
Q_{M P C O R E}=A_{1} \ldots A_{n} U_{1} \ldots U_{n} S_{1} \ldots S_{n} P_{I}\left(S_{1} \ldots S_{n} K_{1}\right) D_{1} \ldots D_{n} \text {, }
$$


where $K_{1}=P_{N}$ in case of the fixed time step method. The algorithm successively allocates the local memory space of the external modules, calls routines $U_{i}$ to fetch the guess values from every module and send them to the external memory space, saves the guess state of the modules using routines $S_{i}$, runs the Picard iteration algorithm $P_{I}$ to get the initial steady-state of the dynamical system, cycles time steps using $K_{1}$ and finally deallocates the external modules.

A time step iteration of the step-doubling method consists of two stages: the first stage performs a time step $h$ to get less accurate solution, the second stage performs two successive time steps of size $h / 2$ to get a more accurate solution. Note that $H_{2}$ is the routine decreasing step $h$ into two times and $H_{1}$ is the routine increasing step $h$ into two times. The one iteration of the step-doubling method is given by

$$
K_{2}=P_{N} H_{2} P_{N} S_{1} \ldots S_{n} P_{N} H_{1}
$$

Time step rejection is an important feature making the multiphysics algorithm more stable to a model fail or the local truncation error (LTE) outbreak due to a large step size. In the case of such a rejection the internal memory spaces of the external models are returned to the previous state and the time step iteration repeats with a smaller step size. The drawback of the algorithms in Equations (7) and (8) is that the save routine $S_{i}$ is called two times in a row for each module. This results in the allocation of additional memory space for the sake of rejection capability. In order to reduce the memory requirements we use the swapped algorithm given by

$$
K_{3}=P_{N} H_{2}\left(L_{1} N_{1} N_{1} U_{1} \ldots L_{n} N_{n} N_{n} U_{n}\right) H_{1} .
$$

Finally, MPCORE employs the time step integration algorithm given by (7) with $K_{1}$ replaced by $K_{3}$. Therefore, the local memory space of the external modules is saved only once per time step iteration and the rejection of the step can be achieved by the loading of external modules from the saved memory space.

Table 1. Exchange of feedback parameters.

\begin{tabular}{lccc}
\hline From/To & RAST-K & FRAPI & CTH1D \\
\hline RAST-K & - & $q_{l}$ & - \\
FRAPI & $T_{\text {fuel }}, T_{\text {clad }}$ & - & $T_{\text {film }}$ \\
CTH1D & $T_{\text {cool }}, D_{\text {cool }}$ & $H_{\text {film }}$ & - \\
\hline
\end{tabular}

\subsection{Step Processing}

MPCORE processes a time step according to algorithm (7) and the scheme shown in Figure 2. For a given reactor core state vector $x$ and time step size $h$ at the local time $t$, the multiphysics code advances the reactor core state from $x$ to $x^{\prime}$ using the step-doubling and Picard iteration methods. The feedback parameters $u_{k}^{\prime}$ from Table 1 are evaluated for the updated solution $x^{\prime}$. The value of LTE $e$, for every time step is evaluated as follows

$$
e=\max _{k \in\{1, \ldots, m\}}\left\|\frac{\Delta_{k}}{A_{t o l}+R_{t o l} u_{k}}\right\|_{2}^{\prime}
$$

where $m$ is the number of feedback parameters, $\Delta_{k}$ is the difference between two solutions obtained with the time steps $h$ and $h / 2 ; A_{t o l}$ and $R_{t o l}$ are the absolute and relative tolerances. For a given LTE update $e^{\prime}$, the new time step is calculated according to the step-doubling theory [13]. Note that the same norm with less relative tolerance $R_{t o l}$ is used for the tracking of fixed-point iteration convergence.

The comparison of the local error, $e^{\prime}$ to 1 results in two logical branches: regular and rejected time steps. For the regular branch, $e^{\prime} \leq 1$, the vector state, local time and step size are updated in order to start 
the next time step iteration. For the rejection branch, $e^{\prime}>1$, the solution $x^{\prime}$ is discarded and the iteration repeats with the smaller step size $h^{\prime}$ computed for the local error $e^{\prime}$. The rejection branch is used for exceptions thrown by the multi-physics code during the Picard iteration. The exception might be thrown by the Picard algorithm itself via the occurrence of either exponential divergence, periodical cycling or slow convergence rate. Moreover, the exception can be thrown by a substituent module that experience the internal divergence or failure event. For the exception of any reason the rejected iteration is repeated using the time size reduced in two times. The rejection may be repeated several times until Picard iteration converges and a small enough local error is achieved. The time step integration algorithm stops with an error if the number of rejecting cycles exceeds the limit or the time step $h^{\prime}$ becomes smaller than the minimum value.

Table 2. Loose coupling interface functions.

\begin{tabular}{lll}
\hline & Name & Description \\
\hline$A$ & alloc & allocation of variables $x$ and $u$ \\
$I$ & init & initial steady-state calculation \\
$N$ & next & moving forward on a time step $h$ \\
$S$ & save & saving of a state vector $x$ \\
$L$ & load & loading of the saved state vector $x$ \\
$U$ & update & update of the feedback vector $u$ \\
$D$ & free & deallocation of the variables \\
\hline
\end{tabular}

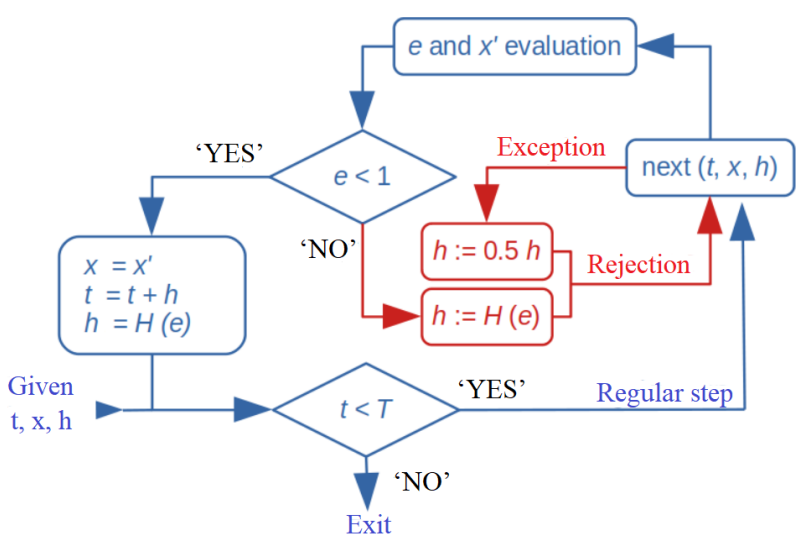

Figure 2. Time step processing flowchart.

\section{Results and Analysis}

MPCORE has been applied for a full-core Pressurized Water Reactor (PWR) Benchmark for Evaluation And Validation of Reactor Simulations (BEAVRS) developed by MIT Computational Reactor Physics Group [26]. The benchmark provides a detailed information about fuel assemblies, burnable absorbers, core loading patterns, in-vessel components and it enables the development and testing of detailed reactor core models for multi-physics calculation. The asymmetric control Rod Ejection Accident (REA) from Hot Zero Power (HZP) for the BEAVRS reactor core is subjected to MPCORE calculation in order to evaluate the impact of dynamic pellet-to-cladding heat transfer on design and safety of LWR.

The purpose of the coupled neutronics, thermal-hydraulics and fuel performance simulation is in more rigorous calculation of the pellet-to-cladding heat transfer to improve fuel rod temperature accuracy. Therefore, in order to evaluate the multi-physics coupling effect, the transient accident is simulated two times starting from the same initial HZP state with a constant and dynamic HTC calculated by Equation (2). 
The following quantities of interest are selected for comparison: peak total core power $W$ in $\mathrm{GW}$, maximum fuel temperature $T_{f c}$ in ${ }^{\circ} \mathrm{C}$, maximum cladding temperature $T_{c i}$ in ${ }^{\circ} \mathrm{C}$, maximum fuel enthalpy $H_{f}$ in cal/g, total energy released to the coolant $Q_{c}$ in GJ and the number of fuel rods with the departure from nucleate boiling (DNB) event $N_{\mathrm{DNB}}$ given in $\%$ of the total number of fuel rods.

\subsection{BEAVRS Core Model}

The RAST-K model of the BEAVRS reactor core uses four radial nodes and 48 axial nodes per fuel assembly for the nodal diffusion calculation. The two-group cross-sections were calculated as the function of fuel temperature, coolant temperature and density, nuclear fuel burnup and boron concentration cross-section code STREAM [27] for each fuel assembly type with and without control rods.

The one-dimensional thermal-hydraulics code uses 20 axial nodes per fuel rod channel, the fuel performance code uses 10 axial nodes, 5 radial nodes in pellet and 3 radial nodes in cladding. The cladding mechanics is calculated by the one-dimensional model FRACAS-I [28], the fission gas release is calculated by FRAPFGR recommended for transient simulation, the fuel grain size is set to $10 \mu \mathrm{m}$. The filling as-fabricated gas is helium, the cladding type is Zircaloy-4, the geometric sizes, initial enrichment and as-fabricated density of the dioxide uranium fuel depend on the location of a fuel pin with respect to the specification of BEAVRS benchmark.

\subsection{HZP and HFP States}

In order to evaluate the accuracy of MPCORE, the beginning of cycle (BOC) hot zero power (HZP) and hot full power (HFP) states have been verified to the benchmark data and to the high-fidelity Monte-Carlo code MCS [29]. The critical boron concentration at the BOC HZP is $937 \mathrm{ppm}$ that is close to the measured value $975 \mathrm{ppm}$. The HZP state was compared to the measurement signals of radial detectors located in the central guide tubes of the fuel assemblies and showing the integral current along core elevation. The numerical signals were approximated by the arithmetic mean of the eight fuel pins neighboring a guide tube. The relative error of the numerical signals in the root-mean-square (RMS) norm accounts for about $7 \%$ while the maximum error reaches 13\%. The HFP assembly-wise radial power distribution compared to MCS demonstrates the RMS relative error about $1 \%$ and $3 \%$ in the maximum. The distribution of errors in the HZP and HFP states are presented in Figure 3. Overall, we conclude the code produces an accurate distribution of power averaged over assembly; however the pin-by-pin distribution error is rather large and further improvement of the RAST-K's reconstruction module is to be undertaken.

\subsection{HZP REA Analysis}

MPCORE calculates a 10-second transient at the BOC to evaluate the behavior of the BEAVRS core in the event of a prompt-critical REA. The simulation starts from the HZP core state with the total power $10^{-6}$ of the nominal power 3411 MWth. The control bank $D$ at the location M-4 in benchmark coordinates has the largest worth $1377 \mathrm{pcm}$ (per cent mille) or $2.0 \beta_{\text {eff }}$ (effective delayed neutron fraction, $1 \beta_{\text {eff }}=705 \mathrm{pcm}$ ) as can be seen in Table 3. This control bank is fully ejected during $100 \mathrm{~ms}$ at a constant rate starting from $10 \mathrm{~ms}$. The transients were calculated for the relative tolerance $0.01 \%$.

The temporal and radial distributions of the safety parameters during the transient are presented in Figures 4 and 5. The reactor core is critical and all the safety parameters are constant before the control bank ejection started at $10 \mathrm{~ms}$. The removal of the neutron absorber from the core produced a reactivity rise up to $2.0 \beta_{\text {eff }}$. When the reactivity reached $1 \beta_{\text {eff }}$ at $50 \mathrm{~ms}$ the reactor core turned prompt-critical and burst with a sharp pulse of neutrons. The total power exponential growth during $104 \mathrm{~ms}$ hit a peak of 43 nominal powers. After the peak the fuel and cladding temperatures responded immediately blowing from initial $293{ }^{\circ} \mathrm{C}$ up to $1300{ }^{\circ} \mathrm{C}$ and $520{ }^{\circ} \mathrm{C}$ respectively. The steep increase in the fuel temperature 
brought down the reactivity from the plateau to about $0.3 \beta_{\text {eff }}$ through the Doppler effect. Therefore, the reactor core switched to delay-critical state and the total power dropped to about $1 \mathrm{GW}$ th according to the amount of delay neutrons in reactor core after the power peak. The maximum fuel temperature grew gradually up to about $1550{ }^{\circ} \mathrm{C}$ during $3 \mathrm{~s}$ after the power peak, the pellet average enthalpy steadily decreased by means of the heat transfer from fuel to the coolant. The excessive heat leaked to the coolant and a high cladding temperature led to a boiling crisis, which was observed in the interval from $0.8 \mathrm{~s}$ to $2 \mathrm{~s}$. The boiling crisis finished owing to the coolant circulation and the decline of the heating of the fuel.

The average gap HTC at the beginning of transient was equal to $5640 \mathrm{~W} /\left(\mathrm{m}^{2} \mathrm{~K}\right)$ for both the dynamic and constant gap HTC cases. The fuel rod heating caused the thermal expansion of both a pellet and cladding materials. However, due to the thermal expansion coefficient difference, the pellet-cladding gap width diminished causing the gas HTC increment in two times with accordance to Equation (4). At the same time the radial HTC increased as well owing to a pellet temperature escalation. Overall, the change of the total gap HTC resulted in the flattening of the cladding and pellet temperatures, as shown in Figure 6. Subsequently, the outer surface cladding temperature for the dynamic gap HTC was larger compared to the constant gap HTC case increasing the likelihood of the DNB event and the number of channels experienced the coolant boiling. The fuel enthalpy, cladding temperature and fuel temperature calculated for both constant and dynamic gap HTC cases are presented in Table 4. Although the values of these parameters are below critical limits, the number of fuel rods with DNB events can violate the acceptance criteria for reactivity accident analysis if dynamic gap HTC model is in use. Note, the similar effect of the dynamic gap HTC has been reported recently for a PWR REA with a $1.3 \beta_{\text {eff }}$ reactivity insertion simulated by the CTF and BISON codes [30].

The results of MPCORE simulation have been verified with the standalone RAST-K code that utilizes a nodal diffusion solver and built-in thermal-hydraulics module to produce the assembly-wise power and temperature distributions. The reactivity, total power, maximum fuel and cladding temperature functions presented in (9) demonstrate the good agreement between two codes with a maximum error about $2 \%$ observed at the power and temperature peaks. The error arises primarily due to the difference in FRAPTRAN's and RAST-K's built-in thermal-hydraulics solvers. Note, the cross-verification of RAST-K and PARCS [31,32] codes implemented for the transient benchmarks NEACRP-PWR [33] and VVER-1000 [34] shows the difference between two codes is less $0.1 \%$. In addition, RAST-K has been used recently for the analysis of rod ejection accident transient in a PWR core with fixed neutron source [35].

(a) MPCORE vs. HZP Detector Signals

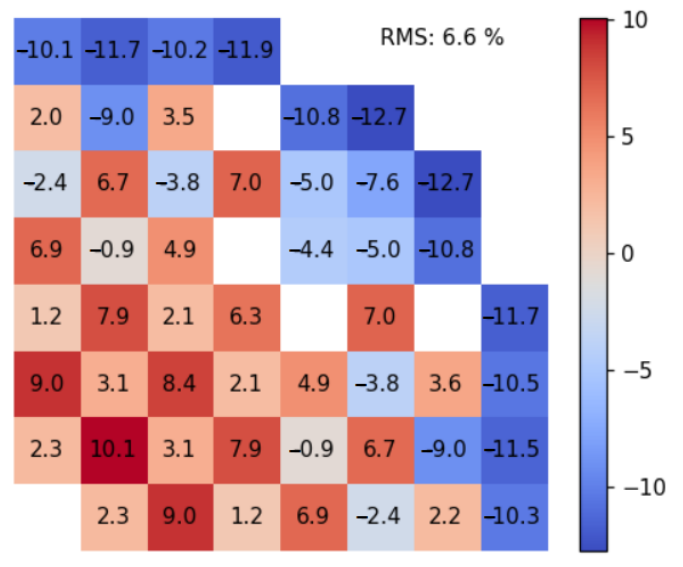

(b) MPCORE vs. MCS HFP Power Distribution

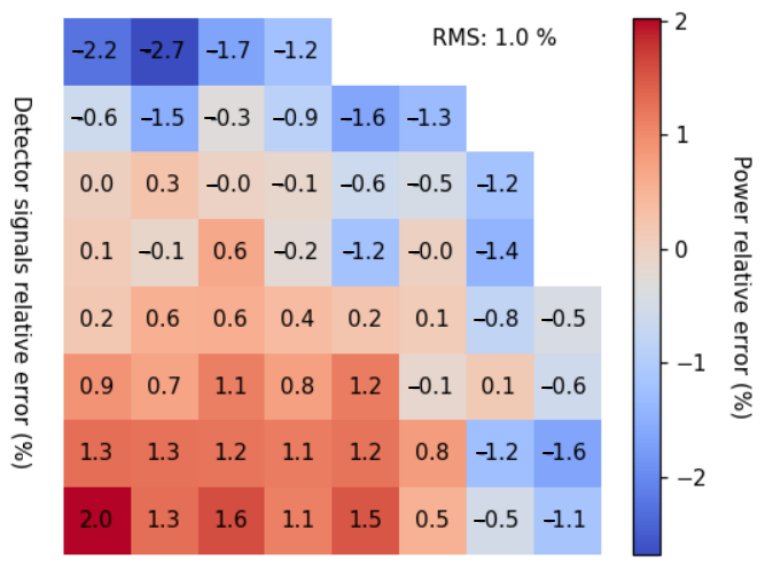

Figure 3. Radial distribution of errors at the beginning of cycle: (a) detector signals compared to measurements; (b) assembly-wise power compared to MCS 
Note, although the assembly-wise distributions for both RAST-K and MPCORE codes are coincide, MPCORE produces more detailed and accurate information owing to the pin-by-pin simulation with the dynamic pellet-to-cladding heat transfer modeling.


Figure 4. Peaking values of the safety parameters as a function of time calculated by MPCORE using dynamic and static gap HTC models, and by RAST-K v.2 with a built-in thermal-hydraulics solver. The solid lines refer to the pin-wise distribution, the dashed lines refer to the assembly-wise distribution. 

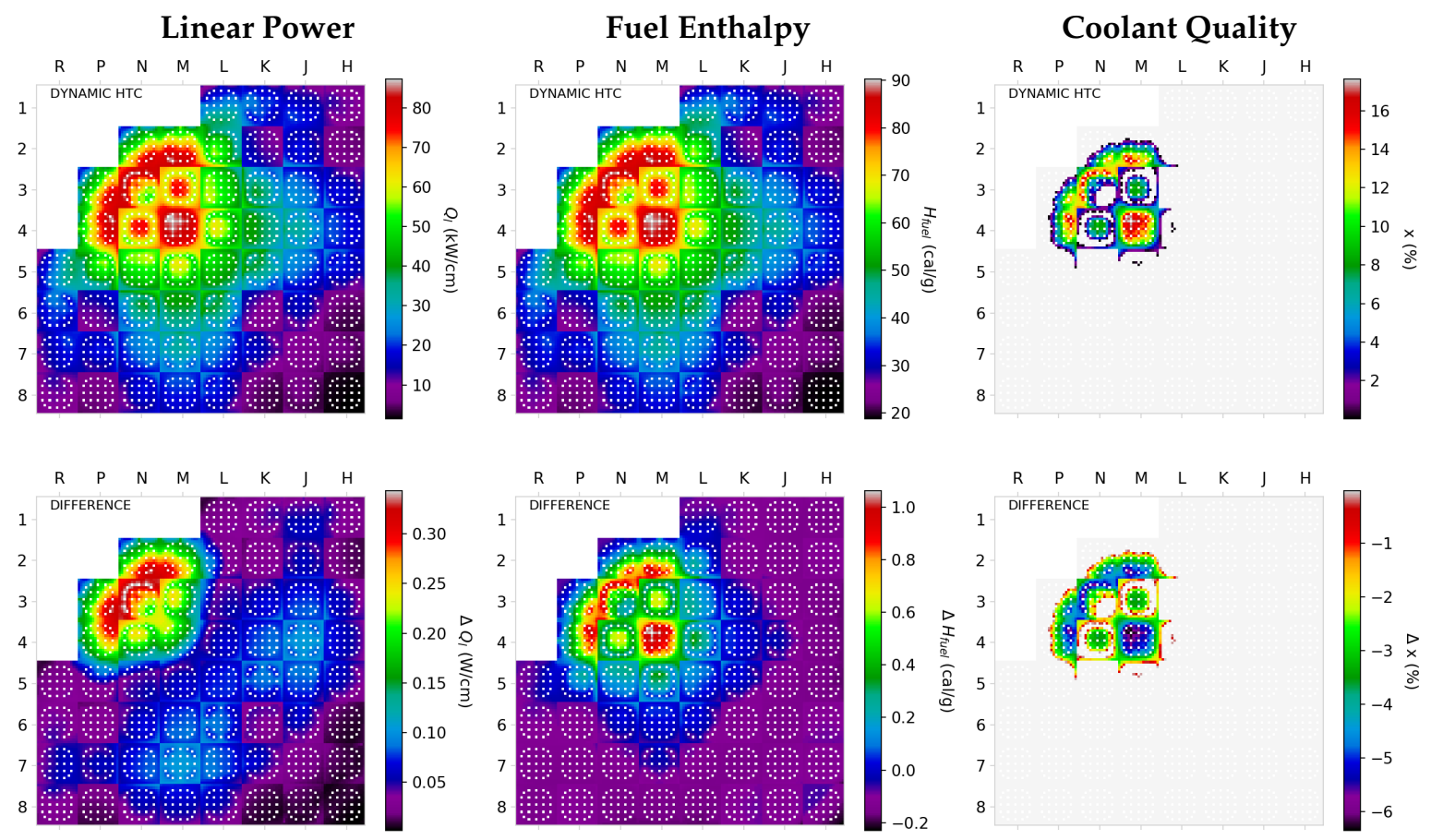

Pellet Temperature

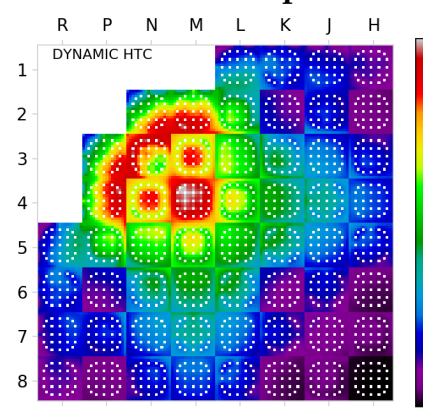

Cladding Temperature

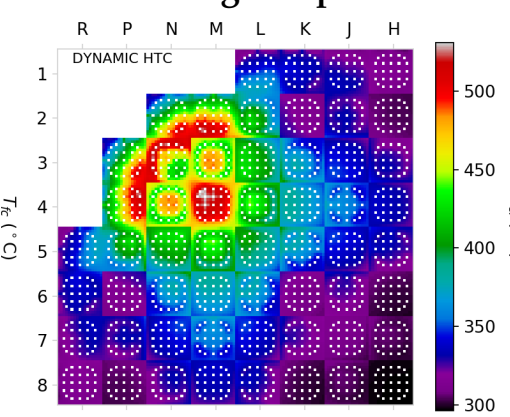

Coolant Temperature

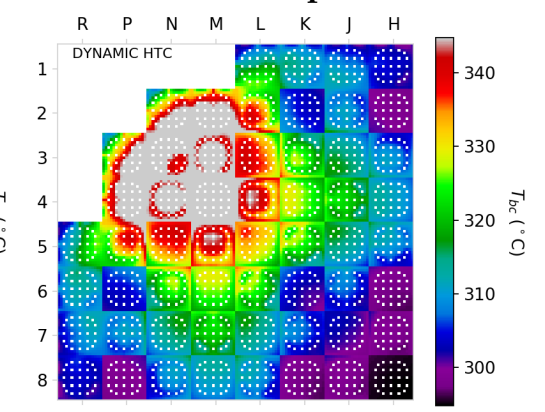

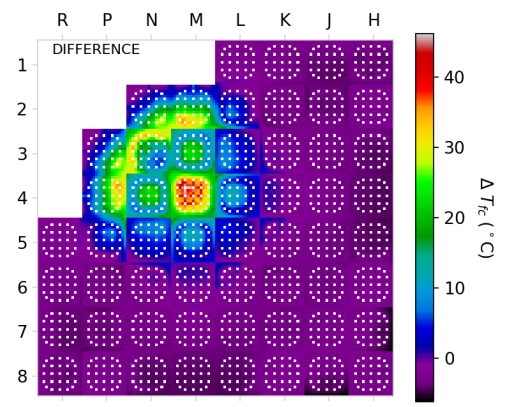
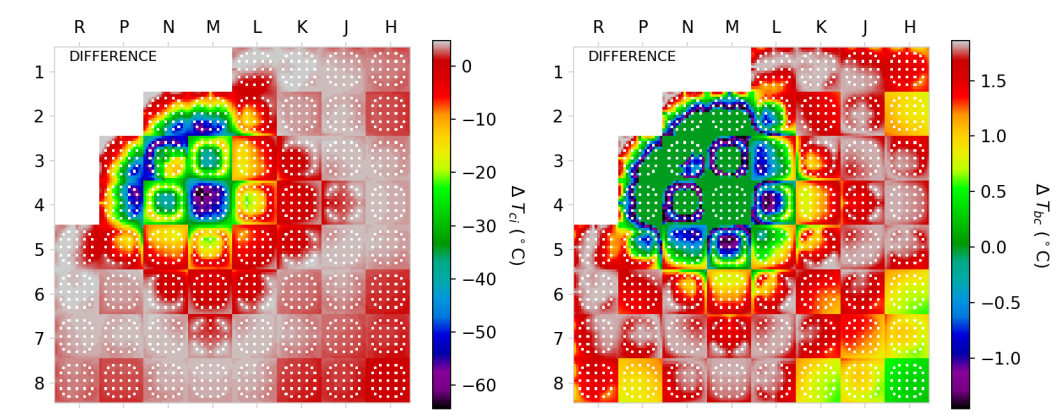

Figure 5. Radial distribution of safety parameters peaks during transient and effect of the pellet-cladding gap HTC dynamical change, simulation tolerance $R_{\text {tol }}=0.01 \%$. 
(a) $T_{f s}-T_{c i}$ Diagram

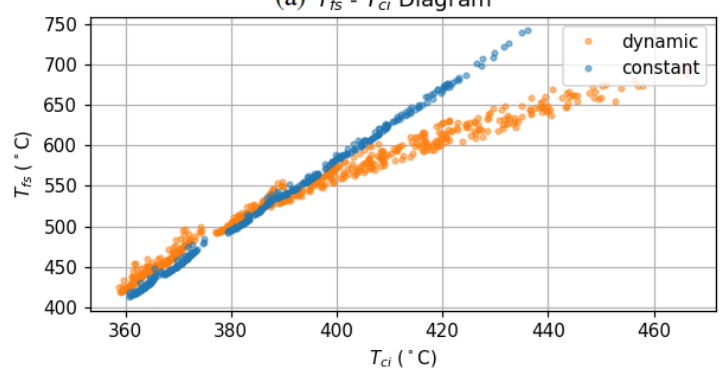

(b) HTC - Gap Size Diagram

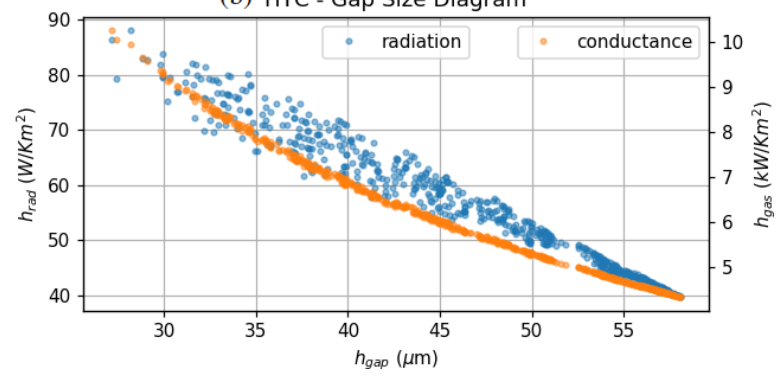

Figure 6. (a) Distribution of pellet and cladding surface temperatures $T_{f_{s}}$ and $T_{c i}$ for the full-core rod ejection accident simulation using dynamic and constant gap heat transfer coefficient models; (b) Distribution of radiation and gas gap heat transfer coefficients as the functions of pellet-cladding gap width for the rod ejection accident simulation using dynamic gap heat transfer coefficient model

Table 3. Worth of control banks.

\begin{tabular}{ccc}
\hline Location & Bank Type & $\boldsymbol{\rho}, \mathbf{p c m}$ \\
\hline H-8 & D & 49 \\
K-8 & A & 175 \\
K-6 & C & 319 \\
H-2 & C & 360 \\
K-2 & B & 589 \\
M-4 & D & 1377 \\
\hline
\end{tabular}

Table 4. Peak safety parameters for constant and dynamic pellet-cladding gap HTC, $R_{t o l}=0.01 \%$.

\begin{tabular}{lcrrrr}
\hline Parameter & Units & Dyn & Const & $\boldsymbol{\Delta}$ & Limits \\
\hline$W t h$ & $G W$ & 147 & 148 & 0 & - \\
$H_{f}$ & $\mathrm{cal} / \mathrm{g}$ & 90 & 91 & 1 & 200 \\
$Q_{c}$ & $M J$ & 564 & 658 & 95 & - \\
$T_{f c}$ & ${ }^{\circ} \mathrm{C}$ & 1543 & 1588 & 45 & 2670 \\
$T_{c i}$ & ${ }^{\circ} \mathrm{C}$ & 531 & 466 & -64 & 1649 \\
$N_{D N B}$ & $\%$ & 2.9 & 2.5 & -0.4 & 5 \\
\hline
\end{tabular}

\subsection{MPCORE Performance}

The convergence of the adaptive time step algorithm was analyzed using one of the BEAVRS' fuel assemblies. The fuel assembly under consideration acquires enrichment $1.6 \%$, coolant flow rate $G=84.1 \mathrm{~kg} / \mathrm{s}$ and initial total power $17.7 \mathrm{~W}$ th; the assembly is modeled with the reflective boundary conditions in the radial directions and zero-current boundary conditions at the assembly top and bottom where reflector is placed. The power pulse was initiated by the reduction of boron concentration from the critical $905 \mathrm{ppm}$ to sub-critical $825 \mathrm{ppm}$ during $10 \mathrm{~ms}$, which is equivalent to the insertion of reactivity $1349 \mathrm{pcm}$. According to RAST-K calculation, conducted for the constant gap HTC $5640 \mathrm{~W} / \mathrm{m}^{2} \mathrm{~K}$ and time step $h=0.1 \mathrm{~ms}$, the power peak of the transient occurs at the time $191.9 \mathrm{~ms}$ and accounts for $2.220 \mathrm{GWth}$; this value was set as a reference for further convergence study.

The fuel assembly was simulated by MPCORE with the constant and dynamic gap HTC models using various relative tolerances $R_{t o l}$ taken between $10^{-3}$ and $1 \%$, while the relative tolerance used for Picard iteration is 100 times less. The graphics of the MPCORE's execution times and power peak errors are presented in Figure 7. The constant and dynamic models show exponential behavior on the relative tolerance; the dynamic case consumes more computation time owing to slower convergence of Picard 
iterations. The relative number of rejected time steps varies between $3 \%$ and $9 \%$. The power peak value converges to approximately the same number for both dynamic and constant gap HTC indicating the initial stage of a REA transient is almost insensitive to the pellet-to-cladding heat transfer simulation.

The analysis of the multi-physics code performance for the full-core case has been done for $R_{t o l}=0.01 \%$, the results are presented in Figure 8. Overall, the simulation completed 363 regular and 55 rejected time steps, 3340 internal iterations and consumed about $42 \mathrm{~h}$ of execution time using one computing processor. The graphic of time step size history shows the minimum step size $1 \mathrm{~ms}$ was at the power peak region; afterwards the step size gradually increased accounting for about $0.5 \mathrm{~s}$ at the end of the simulation interval. The number of function calls per time step iteration was equal to 6 during power pulse; then it increased up to 10 due in response to a thermal-hydraulics model. Some of time steps required over 30 iterations to be converged, which indicates the slow convergence of the Picard iteration applied for the chosen step size. Note, that each iteration includes one large and two small time steps performed according to the step-doubling approach. The number of function calls remains the same for all substituent modules.

(a) Work- $R_{\text {tol }}$ Diagram

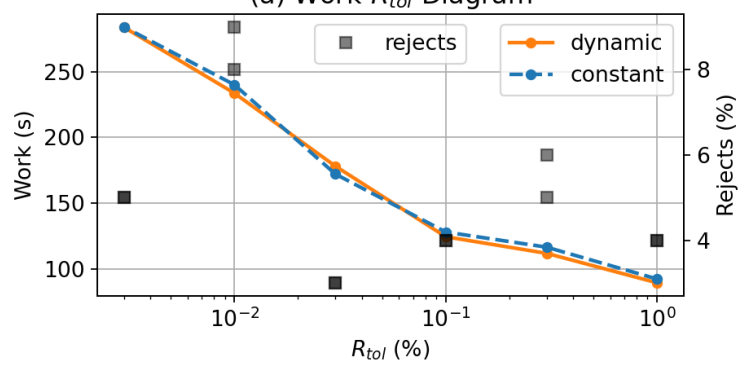

(b) Error- $R_{\text {tol }}$ Diagram

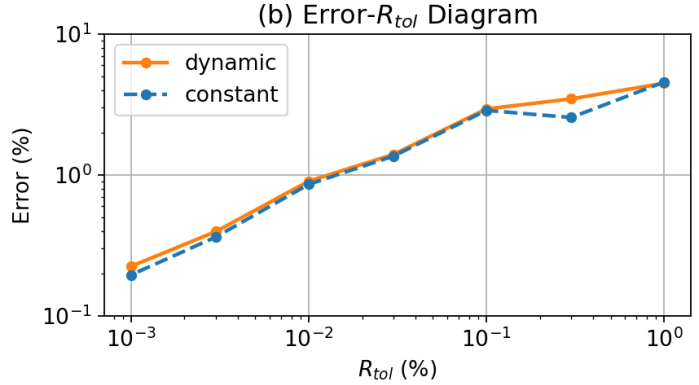

Figure 7. Fuel assembly simulation performance: (a) code run-time and (b) total power peak value error as the functions of relative tolerance $R_{t o l}$.

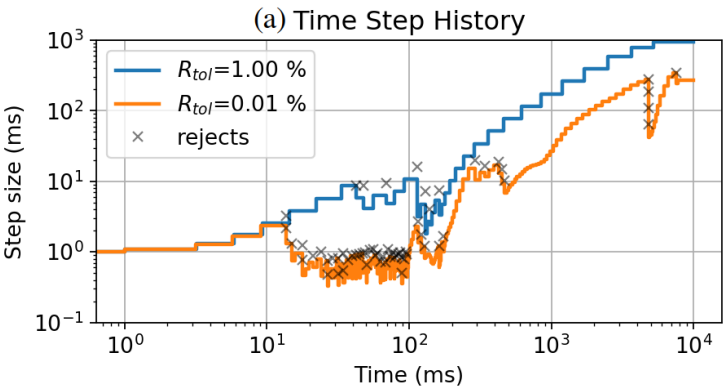

(c) Number of Function Calls $\left(R_{\text {tol }}=0.01 \%\right)$

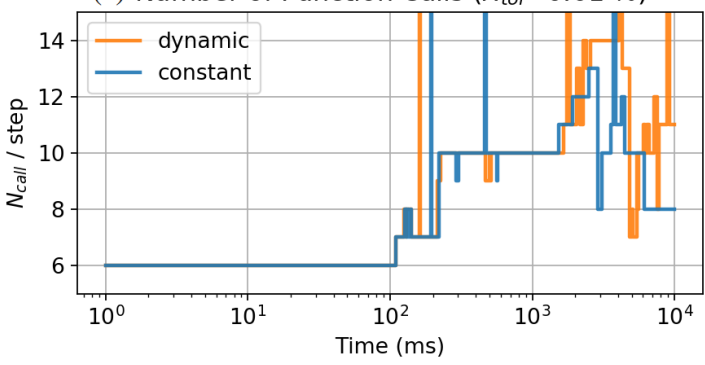

(b) Run Time History $\left(R_{\text {tol }}=0.01 \%\right)$

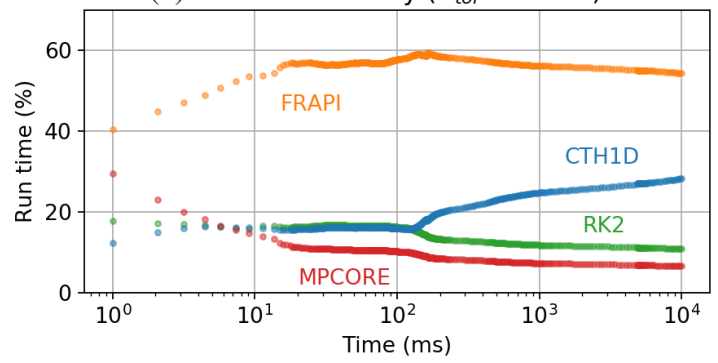

(d) Local Error History $\left(R_{\text {tol }}=0.01 \%\right)$

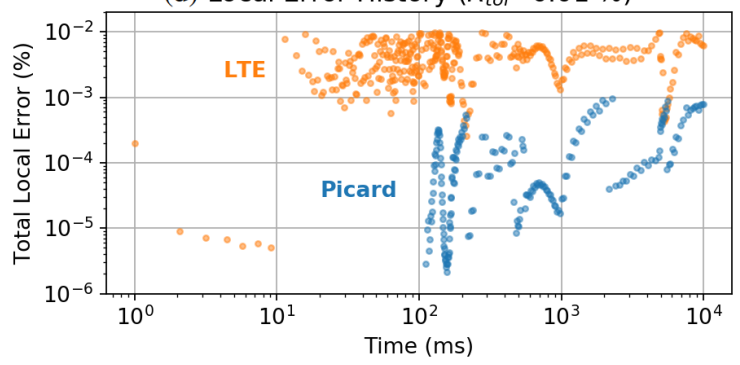

Figure 8. Full-core rod ejection accident performance for relative tolerance $R_{t o l}=0.01 \%$ : (a) time step size, (b) code run-time, (c) function calls number, and (d) local truncation error as the functions of time. 
The most consuming part of the multi-physics simulation is fuel performance that accounts over $50 \%$ of the total execution time. The thermal-hydraulics module requires about $15 \%$ during the calculation of power pulse and $30 \%$ at the end of the simulation interval. The neutronics calculations consume less than $15 \%$ over all time. Note, that MPCORE implements integration of neutron kinetics, thermal-hydraulics and fuel performance modules according to algorithm Equation (7) with the data exchange through a shared memory space. Therefore, the memory-handling routines including $S_{i}, L_{i}, U_{i}$ and damping data routine can be slow as the rank of feedback vectors is large; in this study the overall consumption of the memory-related routine is about $8 \%$.

\section{Conclusions}

The nodal diffusion code RAST-K, fuel performance analysis codes FRAPTRAN, and one-dimensional coolant thermal-hydraulics code CTH1D have been coupled together by means of code MPCORE for the high-resolution multi-physics analysis of transient PWR cores. The new multi-physics system implements the loose coupling of constituent modules enhanced by an adaptive time step algorithm for solution accuracy control and by the restart capability for robustness improvement. Note, each module can be replaced with little efforts on a more suitable or accurate one owing to the modular structure and transparent coupling interface supported by the new coupling system. The present configuration of MPCORE comprised of the two-group nodal diffusion, one-dimensional thermal-hydraulics and thermal-mechanics models is dedicated to the core design optimization and uncertainty quantification where the computational performance is highly demanded for reactor core multiple runs.

The coupled system of multi-physics equations is integrated in time using the step-doubling method to achieve automatic step size and the damped Picard iterations to resolve the non-linear system of equations associated with a given time step. The convergence of the Picard method has been improved by the Gauss-Seidel acceleration algorithm in which a multi-physics module is fulfilled with respect to the feedback information newly updated by a preceding module in the execution chain. Furthermore, the random access memory requirement has been reduced by swapping of the time step and Picard iteration loops in the total coupling algorithm to avoid the allocation of an extra backup memory space needed for the time step restart option.

The new multi-physics system has been applied for the simulation of a hypothetical severe transient accident initiated by ejection of control rods in a PWR core. The BEAVRS benchmark core has been chosen for the test as it provides comprehensive information about fuel pins and assemblies, and enables to develop a detailed multi-physics reactor core model. The simulation was performed in order to assess the accuracy, convergence and performance of MPCORE, as well as in the interest of the effect of dynamic pellet-to-cladding heat transfer modeling on reactor core safety parameters.

Overall, it was found that, while the peaking values of the total core power and the fuel enthalpy are practically indifferent to the choice of constant or dynamic heat transfer models, the impact on the fuel and cladding temperatures is much more considerable. In detail, the increase in the heat transfer coefficient, observed in the dynamic model due to the pellet-cladding gap closing, facilitates the transfer of heat from a fuel pellet to cladding. The rise in the cladding outer surface temperature makes DNB events happen more likely for the larger number of fuel pins. Therefore, the simulation performed using the dynamic gap HTC model can potentially breach the acceptance criteria for reactivity accident analysis, although the fixed gap HTC model produces satisfied results. Note, that the fixed model can be made more conservative using a larger value of HTC than the value fixed at the beginning of transient. However, the conservative HTC value is still unknown and requires either the dynamic heat transfer modeling or an expert's judgment. 
The future work will be focused on the improvement of the inner iterations convergence, and on the development of the cladding-to-fluid heat transfer performance. The full-core simulation reveals the slow convergence of Picard iterations with a fixed damping factor resulting in increase of the rejected time steps number and decrease in a time step size. On the other hand, as was reported in [25], the considerable improvement in convergence rate can be achieved using Anderson's acceleration method that requires a minor modification of the existing iteration scheme. Notwithstanding the relatively slow convergence, the accomplished full-core multi-physics analysis has demonstrated significance of the pellet-to-cladding heat transfer modeling for a severe nuclear accident in a PWR core. However, further investigation of the influence of the model parameters and resolutions on the target reactor core safety characteristics is of vital importance.

Author Contributions: Conceptualization, methodology, A.C.; software, A.C., J.P., H.K. and J.C.; supervision, reviewing, D.L. All authors have read and agreed to the published version of the manuscript.

Funding: This research was supported by National Research Foundation of Korea (NRF) grant funded by the Korea government (MSIT) (No. NRF-2019R1C1C1010063) and partially by the National Research Foundation of Korea (NRF) grant funded by the Korea government (MSIT) (No. NRF-2019M2D2A1A03058371).

Conflicts of Interest: The authors declare no conflict of interest.

\section{Abbreviations}

The following abbreviations are used in this manuscript:

$\begin{array}{ll}\text { BOC } & \text { Beginning of cycle } \\ \text { DNB } & \text { Departure from nucleate boiling } \\ \text { HTC } & \text { Heat transfer coefficient } \\ \text { HFP } & \text { Hot full power } \\ \text { HZP } & \text { Hot zero power } \\ \text { LTE } & \text { Local truncation error } \\ \text { MTU } & \text { Metric ton of uranium } \\ \text { PWR } & \text { Pressurized water reactor } \\ \text { REA } & \text { Rod ejection accident } \\ \text { RMS } & \text { Root mean square } \\ \text { pcm } & \text { Percent mille, } 1 \text { pcm }=10^{-5} \\ \text { ppm } & \text { Parts per million, } 1 \text { ppm }=10^{-6}\end{array}$

\section{References}

1. Wang, J.; Wang, Q.; Ding, M. Review on neutronic/thermal-hydraulic coupling simulation methods for nuclear reactor analysis. Ann. Nucl. Energy 2020, 137, 1377-1386. [CrossRef]

2. Ivanov, K.; Avramova, M.N. Challenges in coupled thermal-hydraulics and neutronics simulations for LWR safety analysis. Ann. Nucl. Energy 2007, 34, 501-513. [CrossRef]

3. Yu, J.; Lee, H.; Lemaire, M.; Kim, H.; Zhang, P.; Lee, D. MCS based neutronics/thermal-hydraulics/fuelperformance coupling with CTF and FRAPCON. Comput. Phys. Commun. 2019, 238, 1-18. [CrossRef]

4. Kim, W.; Lee, H.; Lemaire, M.; Kim, H.; Zhang, P.; Lee, D. Fuel performance analysis of BEAVRS benchmark Cycle 1 depletion with MCS/FRAPCON coupled system. Ann. Nucl. Energy 2020, 138, 107192.

5. Jang, J.; Kim, W.; Jeong, S.; Jeong, E.; Park, J.; Lemaire, M.; Lee, H.; Jo, Y.; Zhang, P.; Lee, D. Validation of UNIST Monte Carlo Code MCS for Criticality Safety Analysis of PWR Spent Fuel Pool and Storage Cask. Ann. Nucl. Energy 2018, 114, 495-509. [CrossRef]

6. Salko, R. CTF Theory Manual; Reactor Dynamics and Fuel Management Group, Pennsylvania State University: State College, PA, USA, 2014. 
7. Geelhood, K.; Lusher, W. FRAPCON-4.0: Integral Assessment; Pacific Northwest National Laboratory: Richland, WA, USA, 2015.

8. Cherezov, A.; Jang, J.; Lee, D. MPCORE Code for OPR-1000 Transient Multiphysics Simulation with Adaptive Time Step Size Control. In Proceedings of the American Nuclear Society Annual Meeting, Miami Beach, FL, USA, 8-11 June 2020; pp. 389-392.

9. Cherezov, A.; Jang, J.; Lee, D. A PCA compression method for reactor core transient multiphysics simulation. Prog. Nucl. Energy 2020, 128, 103441. [CrossRef]

10. Jernkvist, L.; Massih, A. State-of-the-Art Report. Nuclear Fuel Behavior under Reactivity-Initiated Accident (RIA) Conditions; Nuclear Energy Agency, OECD: Paris, France, 2010.

11. Rudling, P.; Jernkvist, L.O.; Garzarolli, F.; Adamson, R.; Mahmood, T.; Strasser, A.; Patterson, C. Nuclear Fuel Behavior under RIA Conditions; Advanced Nuclear Technology International Europe AB: Tollered, Sweden, 2016.

12. Panka, I.; Kereszturi, A.; Molnar, A. Coupling the Fraptran Fuel Behavior and the TRABCO Hot Channel Thermal Hydraulics Codes. In Proceedings of the 21st AER Symposium, Dresden, Germany, 23 September 2011.

13. Shampine, L. Local error estimation by doubling. Computing 1985, 34, 179-190. [CrossRef]

14. Hairer, E. Solving Ordinary Differential Equations: I. Nonstiff Problems; Springer: Berlin/Heidelberg, Germany, 1987.

15. Zimin, V.G.; Cherezov, A. Application of Backward Differentiation Formulas to Neutron Kinetics Problems. Phys. At. Nucl. 2017, 80, 1377-1386. [CrossRef]

16. Choe, J.; Choi, S.; Zhang, P.; Park, J.; Kim, W.; Shin, H.C.; Lee, H.C.; Jung, J.E.; Lee, D. Verification and validation of STREAM/RAST-K for PWR analysis. Nucl. Eng. Technol. 2019, 51, 356-368. [CrossRef]

17. Geelhood, K.; Luscher, W.C.; Cuta, J.M. FRAPTRAN-2.0: A Computer Code for the Transient Analysis of Oxide Fuel Rods; Pacifc Northwest National Laboratory: Richland, WA, USA, 2016.

18. Cherezov, A. Fuel Rod Analysis Programming Interface for a Loosely Coupled Multiphysics System. In Proceedings of the American Nuclear Society Annual Meeting, Chicago, IL, USA, 16-19 November 2020; pp. 1331-1334.

19. Cacuci, D. Handbook of Nuclear Engineering; Springer: Berlin/Heidelberg, Germany, 2010.

20. Lassmann, K.; Hohlefeld, F. The revised URGAP model to describe the gap conductance between fuel and cladding. Nucl. Eng. Des. 1987, 103, 215-221. [CrossRef]

21. Ross, A.; Stoute, R. Heat Transfer Coefficient between UO2 and Zircaloy-2; CRFD-1075; Atomic Energy of Canada Limited: Chalk River, ON, Cananda, 1962.

22. Trott, D.; Rader, D.; Castaneda, J.; Torczynski, J.; Gallis, M. Experimental Measurements of Thermal Accommodation Coefficients for Microscale Gas-Phase Heat Transfer. In Proceedings of the 39th AIAA Thermophysics Conference, Session: TP-6: Thermophysical Properties, Reno, NV, USA, 8-12 January 2007.

23. D'Auria, F.D.; Reventos, F.; Sjoberg, A.; Sandervag, O.; Anhert, C.; Verdu, G.; Hadek, J.; Ivanov, K.; Uddin, R.; Sartoti, E.; et al. Neutronics/Thermal-hydraulics Coupling in LWR Technology: State-of-the-Art Report (REAC-SOAR); OECD NEA 1734/01; OECD: Paris, France, 2004.

24. Choe, J.; Sooyoung, C.; Jinsu, P.; Hyunsuk, L.; Matthieu, L.; Cheol, S.H.; Soo, L.H.; Deokjung, L. Verification of Asymmetric Fuel Assembly Treatment in STREAM/RAST-K 2.0. In Proceedings of the International Conference on Mathematics and Computational Methods Applied to Nuclear Science and Engineering, Portland, OR, USA, 25-29 August 2019.

25. Hamilton, S.; Berrill, M.; Clarno, K.T.; Pawlowski, R.; Toth, A.; Kelley, C.T.; Evans, T.M.; Philip, B. An assessment of coupling algorithms for nuclear reactor core physics simulations. J. Comput. Phys. 2016, 311, $241-257$. [CrossRef]

26. Horelik, N. MIT Benchmark for Evaluation and Validation of Reactor Simulations (BEAVRS), Version 2.0; MIT Computational Reactor Physics Group, Massachusetts Institute of Technology: Cambridge, MA, USA, 2016.

27. Choi, S.; Lee, C.; Lee, D. Resonance Treatment using Pin-Based Pointwise Energy Slowing-Down Method. J. Comput. Phys. 2017, 330, 134-155. [CrossRef]

28. Bohn, M. FRACAS: A Subcode for the Analysis of Fuel Pellet-Cladding Mechanical Interaction; Idaho National Engineering Laboratory: Idaho Falls, ID, USA, 1977. 
29. Lee, H.; Kim, W.; Zhang, P.; Lemaire, M.; Khassenov, A.; Yu, J.; Jo, Y.; Park, J.; Lee, D. MCS—A Monte Carlo Particle Transport Code for Large-Scale Power Reactor Analysis. Ann. Nucl. Energy 2020, 139, 107276. [CrossRef]

30. Wysocki, A.; Hu, J.; Salko, R.; Kochunas, B. Improvement of CTF for RIA Analysis. In Proceedings of the American Nuclear Society Winter Meeting, (Public Access through US Department of Energy), Nashville, TN, USA, 16-19 November 2020.

31. Downar, T.; Xu, Y.; Seker, V.; Hudson, N. PARCS v3.0 U.S.NRC Core Neutronics Simulator User Manual; UM-NERS-09-0001; Purdue University: West Lafayette, IN, USA, 2010.

32. Diamond, D.; Bromley, B.P.; Aronson, A.L. Studies of the Rod Ejection Accident in a PWR; Brookhaven National Laboratory: Upton County, TX, USA, 2002.

33. Jeong, E. Verification and Validation for Transient Calculation of STREAM/RAST-K 2.0. Master's Thesis, Department of Nuclear Engineering, Graduate School of UNIST, Ulsan, Korea, 2018; p. 46.

34. Jang, J.; Cherezov, A.; Jo, Y.; Lee, D. Verification of hexagonal transient solver implemented in RAST-K with OCED/NEA benchmark problem of KALININ-3 NPP. In Proceedings of the Transactions of the Korean Nuclear Society Autumn Meeting, Changwon, Korea, 22-23 October 2020.

35. Jo, Y.; Shin, H. Low power transient analysis of PWR reload core with fixed neutron source via 3-D nodal diffusion code RAST-K. Ann. Nucl. Energy 2020, 150, 107742. [CrossRef]

Publisher's Note: MDPI stays neutral with regard to jurisdictional claims in published maps and institutional affiliations.

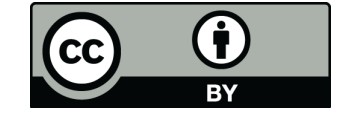

(C) 2020 by the authors. Licensee MDPI, Basel, Switzerland. This article is an open access article distributed under the terms and conditions of the Creative Commons Attribution (CC BY) license (http:/ / creativecommons.org/licenses/by/4.0/). 